\title{
DEFORMATION AND TEXTURE EVOLUTION OF A 3004 ALUMINIUM ALLOY DURING IRONING
}

\author{
F. HEYMÈS ${ }^{* *}$, J. D. EMBURY*, R. SOWERBY* and U. F. KOCKS ${ }^{+}$ \\ ** Pechiney CRV, Voreppe, France. \\ * McMaster University, Hamilton, Ontario, Canada. \\ + Los Alamos National Laboratories, Los Alamos, New Mexico, USA.
}

(Received 20 February 1995; in final form 14 May, 1995)

\begin{abstract}
The production of drawn and ironed (D\&I) beverage cans represents an important example of process optimisation. The aluminium alloy $3004 \mathrm{H} 19$ is widely used for the can body, since it has a good combination of formability and strength and results in very little earing after deep drawing. These desirable properties are achieved by exercising careful control of the rolling schedule of the can stock sheet. During the ironing stage the wall thickness is reduced by a factor of about three, and these large strains determine the resulting texture in the can wall. Large shear strains are also present and these can vary through the wall thickness. The present work deals with the deformation and texture evolution when ironing strips of 3004 aluminium. The strip material was in either a cold rolled or an annealed condition. The texture on each surface of the ironed strips, as well as at the mid-plane, was determined experimentally. The experimental results were then compared with the results from a computer program which was capable of predicting the texture evolution.
\end{abstract}

KEY WORDS Beverage cans, Ironing, Aluminium Alloys, Texture, Orientation Distribution Functions.

\section{EXPERIMENTAL PROCEDURE}

\subsection{Ironing Experiments}

In a typical production line the can body is first drawn and then redrawn to produce a cylindrical cup, which is then pushed through a number (usually three) of ironing dies which reduce the wall thickness and at the same time increase the length of the can body. The initial thickness of the can stock is about $0.33 \mathrm{~mm}$, and this does not change much in the draw and redraw stages. However, during the ironing phase the wall thickness is reduced to about $0.12 \mathrm{~mm}$, the overall reduction is shared approximately equally in each of the ironing dies.

In the present study the actual ironing process is simulated using the equipment shown in Figure 1, which can be mounted in a Universal Testing machine. With this apparatus strips of material are ironed in a plane strain (no change in width) mode, thus providing a two dimensional analogue to the axi-symmetric process. A wedge shaped die was impressed into the strip in the through thickness direction to set the reduction. This was controlled by a cam, where the amount of rotation fixed the depth of penetration and hence the reduction. Resistance to the force of penetration was provided by a fixed rectangular bar against which the strip was pressed. The bar, referred 


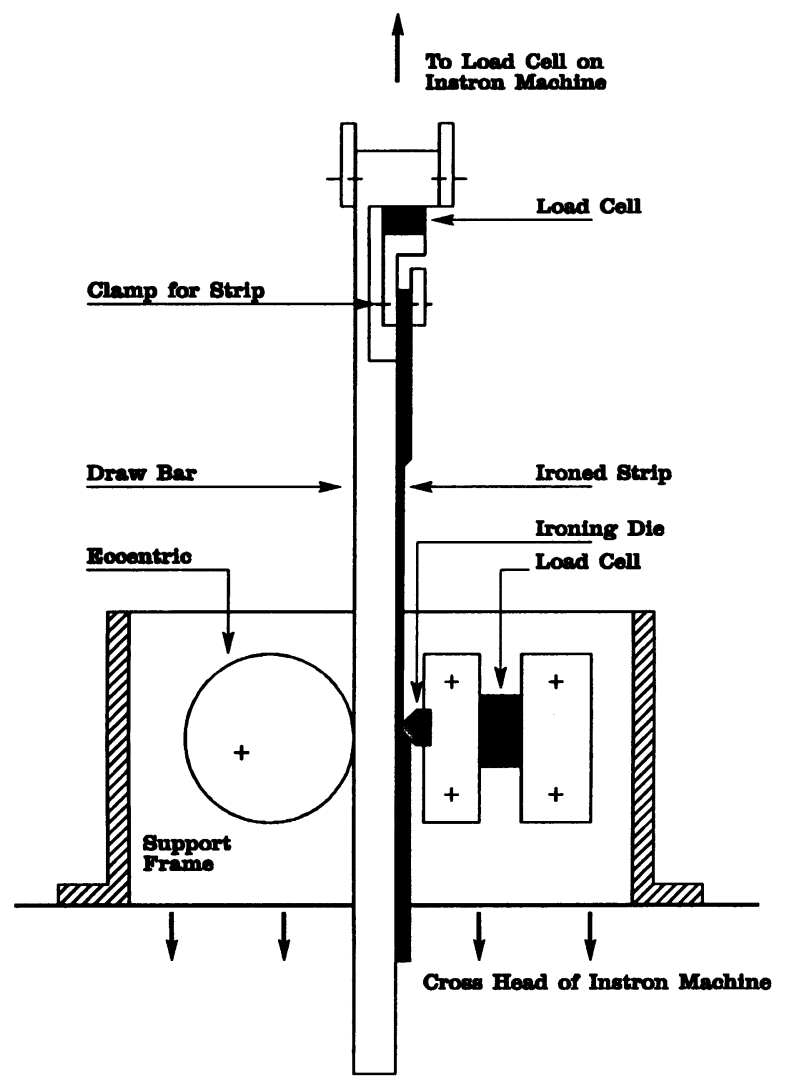

Figure 1 The strip ironing device.

to as the draw bar, is visible in Figure 1 and plays the role of the punch or ram in the actual ironing operation.

Once locked in position at a set reduction the wedged shape die is moved down over the surface of the strip which is therefore elongated and moves relatives to the fixed draw bar. The ironing device was equipped with a number of load cells in order to monitor the various forces present in the ironing operation. It was then possible to calculate the average coefficient of friction acting between the strip and the die and the strip and the draw bar.

Figure 2 is a schematic representation of the ironing operation, and the operative stresses are depicted in the figure. Further details of the ironing dynamometer are to be found in Heymès (1992).

In the ironing experiments the reduction was set to about $30 \%$ which is similar to the reduction achieved in each ironing die in the actual operation. To simplify the metallographic study, a $0.7 \mathrm{~mm}$ thick sheet was used rather than the actual can stock which is $0.33 \mathrm{~mm}$ thick. The sheet employed was a 3004 aluminium alloy, in the H14 temper as opposed to the fully hardened H19 temper. The difference in the two cold rolling tempers has little influence on the texture, the H19 temper contains a slightly stronger copper rolling texture. The H14 temper material is referred to henceforth as 


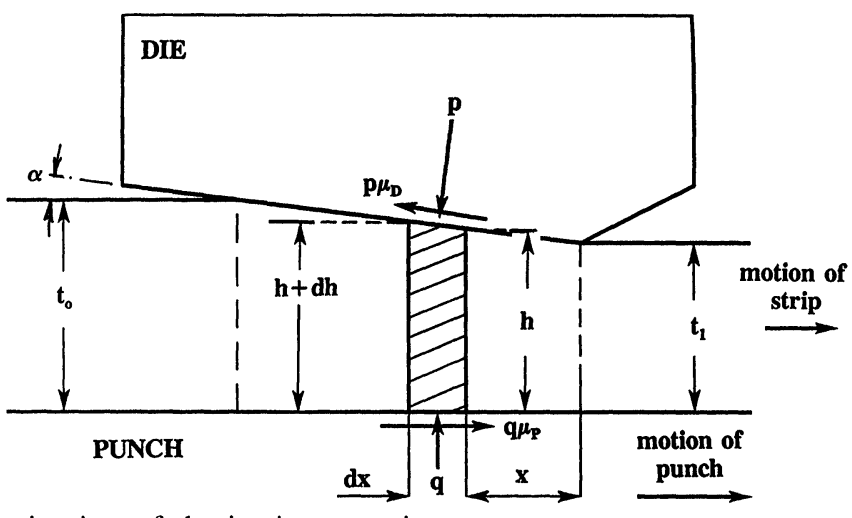

Figure 2 Schematic view of the ironing operation.

material P2. A SEM photograph of the microstructure is shown in Figure 3. The fine lamellar surface relief shown in Figure 3 was obtained using an Anodic Film technique described by Hone and Pearson (1950). The chemical composition of the material is shown in the Table 1.

Experiments were also performed on the 3004 material but in the fully annealed condition i.e. annealed for 3 hours at $300^{\circ} \mathrm{C}-$ this material is designated as $P 2 R$. It should be noted that no grain growth was observed for holding times between $1 / 2$ and

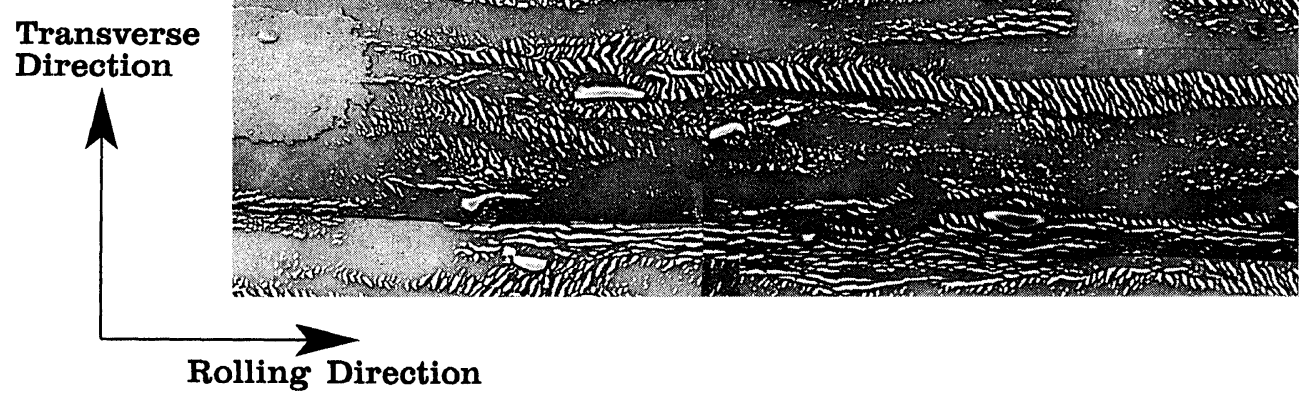

Figure 3 SEM photograph of the surface of the cold rolled material P2 (x1200).

Table 1 Weight Percentage of the Major Alloys in the P2 Material

\begin{tabular}{llllll}
\hline Material & $M n$ & $M g$ & $F e$ & $S i$ & $C u$ \\
\hline P2/P2R & 0.79 & 1.12 & 0.38 & 0.18 & 0.11 \\
\hline
\end{tabular}

The elements $\mathrm{Cr}, \mathrm{Ni}$ and $\mathrm{Zn}$ were found as traces at less than $0.01 \%$ by weight. 


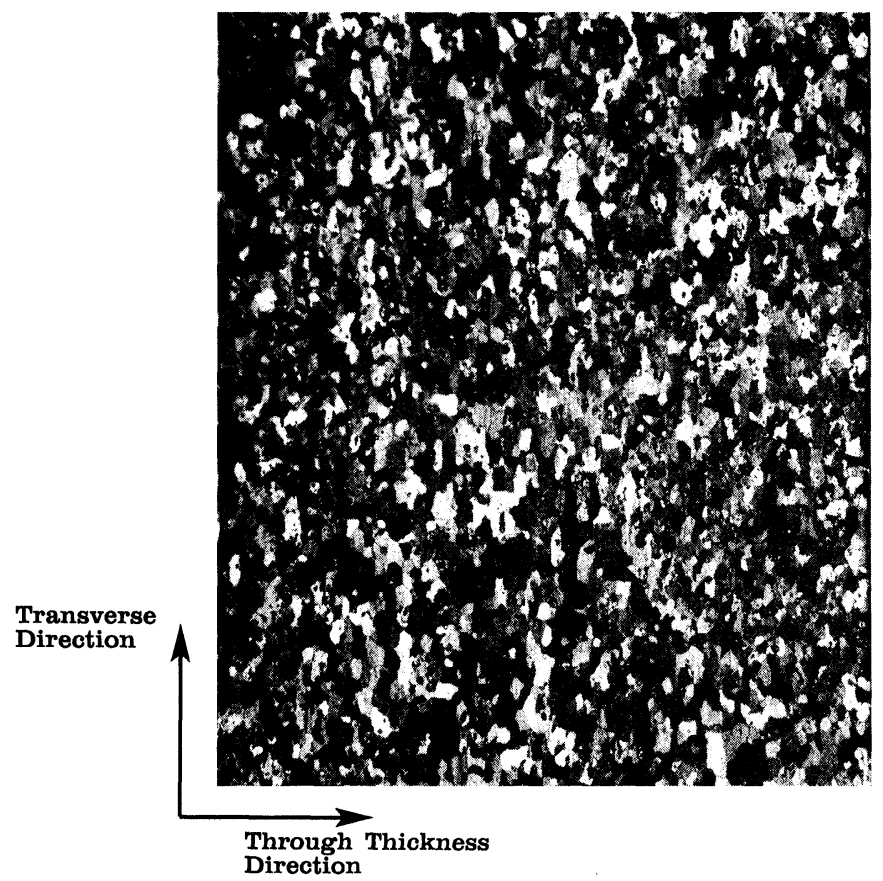

Figure 4 Photomicrograph of the through thickness of the recrystallized material P2R along the transverse direction $(\mathrm{x} 130)$.

5 hours, and this is attributed to the homogeneous dispersion of fine $M n$ precipitates. The heat treatment resulted in a regular equiaxed grain structure, and this is evident from Figure 4 which is a micrograph of the through thickness of the annealed strip along the transverse direction. The resulting grain size for both the $P 2$ and $P 2 R$ materials prior to ironing is summarized in Table 2 , the mean grain size is given in $\mu \mathrm{m}$ for different orientations.

Strip samples were cut along the transverse direction from both the P2 and PR2 materials. The strips were nominally $15.5 \mathrm{~mm}$ wide and $0.6 \mathrm{~m}$ long. It was considered that ironing along the transverse direction, rather than along the original rolling direction of the material, would result in a more dramatic change in texture.

\subsection{Measuring the Deformation}

In order to predict how the texture develops it is necessary to know the strain history. To monitor the metal flow during ironing, a series of micro-hardness indentations were

Table 2 Grain Size of the Cold Rolled Material (P2) and the Recrystallized Material (P2R)

\begin{tabular}{llll}
\hline $\begin{array}{l}\text { Grain Size } \\
(\mu \mathrm{m})\end{array}$ & $\begin{array}{l}\text { Rolling } \\
\text { Direction }\end{array}$ & $\begin{array}{l}\text { Transverse } \\
\text { Direction }\end{array}$ & $\begin{array}{l}\text { Through } \\
\text { Thickness }\end{array}$ \\
\hline P2 & 300 & 60 & 4 \\
P2R & $20-40$ & $20-40$ & $10-20$ \\
\hline
\end{tabular}


made across the thickness of a sample before ironing. The indentation marks experience a compression in the through thickness direction, as well as an imposed shear. The shearing arises because of the presence of friction and also because the material is being deformed through wedge shaped ironing dies, which will also induce inhomogeneous deformation. The amount of shear strain can be estimated from the displacement of the indentation marks. Since the actual deformation mode is not known, i.e. only the initial and final configuration of the indentation marks are known but not the actual deformation path, then some assumption must be made about the deformation path. Such a method has been proposed by Sowerby et al (1986), where a pure homogeneous mode was assumed i.e. no rotation of the principal strain axes with respect to the material element during deformation. It should be noted that this assumption is likely to underestimate the actual representative strain that is incurred in the process, see Sowerby et al. (1986) and Sowerby and Chakravarti (1983) for a fuller discussion.

In order to estimate the shear strain it is further assumed that the indentation marks remain collinear after deformation, this is a prerequisite of a homogeneous deformation mode i.e. straight lines are mapped into straight lines. The deformation is characterized through the deformation gradient tensor $\boldsymbol{F}$, whose components can be determined for the ironing operation.

This can be explained with reference to Figure 5 where $O A$ and $O B$ represent a pair of orthogonal lines marked in a plane which comprises the thickness and transverse directions respectively of the undeformed strip. These directions can also be taken to correspond to a set of coordinate axes, oy and $o x$ respectively. For example, the indentation marks would lie along a line such as $O A$. Hence the length of $O A$ is known since it covers a number of indentation marks whose distance apart was measured at the time they were made. After deformation $O A$ moves to $O A^{\prime}$ and $O B$ to $O B^{\prime}$, which

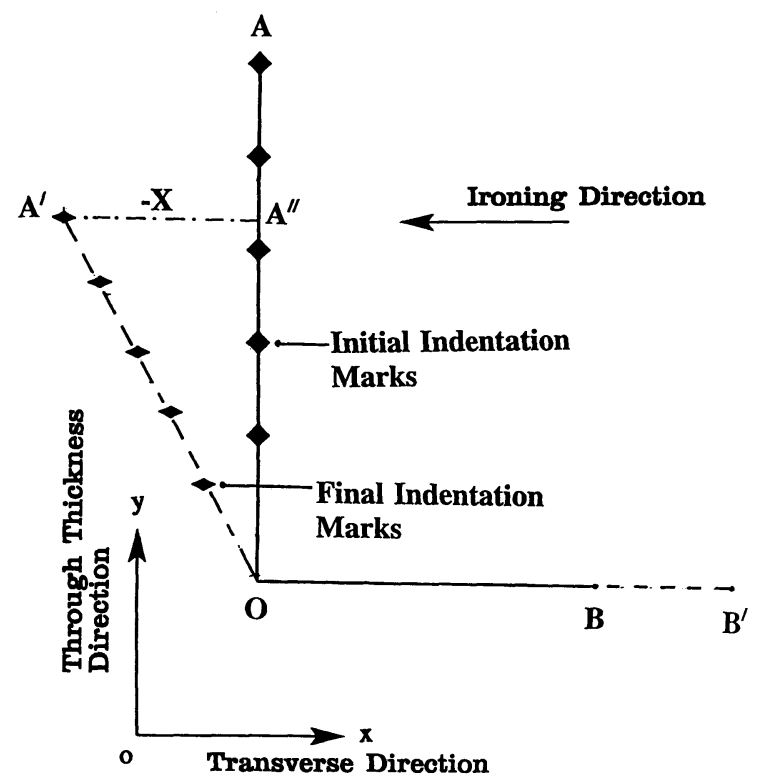

Figure 5 Illustration of the assumed deformation mode in the ironing process. 
depicts a shearing process. Now project $O A^{\prime}$ onto oy to give $O A^{\prime \prime}$, and the ratio $O A^{\prime \prime} / O A$ is given by

$$
O A^{\prime \prime} / O A=t / t_{o}=1 / R
$$

where $t_{o}$ is the original thickness of the strip and $t$ the thickness after ironing. Since the process is assumed to be plane strain then $O B^{\prime} / O B=t_{d} / t=R$. As can be seen from Figure 5, one shear component, say $S$, is given by $S=-X / O A$ (shearing in the negative sense of the $x$-coordinate), while the other shear component is zero. Hence the components of the deformation gradient tensor, $F$, are given by

$$
\boldsymbol{F}=\left|\begin{array}{ll}
F_{x x} & F_{x y} \\
F_{y x} & F_{y y}
\end{array}\right|=\left|\begin{array}{cc}
R & -S \\
0 & 1 / R
\end{array}\right|
$$

The above tensor is clearly unsymmetric, and in order to make a symmetric tensor the following manipulation is invoked, see Sowerby et al. (1985) for the details, let

$$
\boldsymbol{C}=\boldsymbol{F}^{\boldsymbol{T}} \boldsymbol{F}=\left|\begin{array}{ll}
C_{x x} & C_{x y} \\
C_{y x} & C_{y y}
\end{array}\right|
$$

In the above expression, $\boldsymbol{F}^{T}$ is the transpose of $\boldsymbol{F}$, and the resulting matrix multiplication yields a symmetric tensor $\boldsymbol{C}$, named the Cauchy-Green deformation tensor. It is now possible to find the eigenvalues or the principal values of the tensor quantity, this is a standard procedure. The principal elongation ratios squared are then given by,

$$
\lambda_{11}^{2}, \lambda_{22}{ }^{2}=\frac{C_{x x}+C_{y y}}{2} \pm \sqrt{\left\{\frac{C_{x x}-C_{y y}}{2}\right\}^{2}+C_{x y}{ }^{2}}
$$

The principal natural strains are $\varepsilon_{11}=\operatorname{In} \lambda_{11}$ and $\varepsilon_{22}=\operatorname{In} \lambda_{22}$.

The orientation of the maximum principal stretch axis measured anticlockwise with respect to the $x$-axis, see Figure 5 , is given by

$$
\tan (2 \theta)=2 C_{x y} /\left(C_{x x}-C_{y y}\right) \text {. }
$$

In the present work it turned out that the angle $\theta$ was small and therefore it is possible to obtain an estimate of the strains referred to the $x-y$ coordinate axes. This is achieved by assuming

$$
e_{x y} / e_{x x} \approx \tan (2 \theta)
$$

and that

$$
e_{x x}=-e_{y y}=\operatorname{In} R \text {. }
$$

It is emphasised that the above calculation is performed to obtain an estimate of the shear strain only. As mentioned in the next section the texture evolution is predicted by computer programs developed at the Los Alamos National Laboratories. 


\subsection{Texture Measurements}

Texture measurements were performed on strips of the cold rolled $(P 2)$ and annealed $(P 2 R)$ materials prior to ironing. The measurements were taken on the outside surface and also at the mid plane. For the ironing experiments strips of both the cold rolled and annealed material were cut transversely to the original rolling direction. After ironing texture measurements were made on each surface of the strips as well as at the mid-plane.

All samples were cut with a low speed saw and the edges and corners ground to obtain a flat surface. To prepare for texture measurements on the mid plane, the thickness was first reduced by grinding and then removing $100 \mu \mathrm{m}$ by polishing. The polishing was done successively using $6 \mu \mathrm{m}, 1 \mu \mathrm{m}$ and $0.3 \mu \mathrm{m}$ grit wheels, in order to minimise the work hardened layer due to grinding. When taking the texture measurements on the surface of the ironed strip which had been in contact with the die, no polishing was performed in order to avoid the removal of the thin work-hardened layer. This region was of particular interest, and for these samples polishing was not necessary since the surface finish was usually very good. For the surface which had been in contact with the draw bar, light polishing with the $1 \mu \mathrm{m}$ diamond and $0.3 \mu \mathrm{m}$ alumina wheels was performed. A final suface preparation was then performed on all the samples after polishing, by etching in a solution of nitric acid, ethanol and hydrogen peroxide for one minute.

The texture data were obtained by first measuring the incomplete pole figures (111), (200), (220) and (311) using a goniometer with a $\mathrm{Cu} \mathrm{K \alpha} \mathrm{X}$-ray source. The data were corrected for defocusing and background intensity and were analyzed by the texture analysis package, popLA, described by Kallend et al. (1992). The Big WIMV algorithm was then used to calculate from the (111), (200) and (220) pole figures, the $O D F$ (orientation distribution function). When calculating the $O D F$ for the $P 2$ and $P R 2$ materials prior to ironing, orthotropic sample symmetry was assumed, in addition to the cubic crystal symmetry.

The software package popLA also includes a program referred to as LApp, see Kocks et al. (1988), which calculates the texture evolution arising from any imposed deformation mode and boundary conditions. The program was used to simulate the texture change in the ironing process, orthotropic symmetry was no longer assumed in these calculations. The measured texture before ironing was discretized in the form of a weights file of 504 grains. For the cold rolled sheet the rolling axis was not imposed as a symmetry axis. Initially, the texture evolution was modelled using a full-constraints simulation. The weights files developed from the simulation were then converted to pole figures, which were then smoothed with a Gaussian filter with a $5^{\circ}$ decay distance which represents the experimental step angle.

At a later date some additional calculations were performed using a relaxed constraints simulation (with $7.5^{\circ}$ smoothing) which could account for the grain aspect ratios, and thus provided an interesting comparison with the full constraints simulation.

It was decided to present the results in the form of pole figures, because it was felt this provided a clear comparison of the effect of either including or ignoring the influence of the shear strain component in the texture simulation. All the pole figures presented are in the form of stereographic projection. 


\section{RESULTS AND DISCUSSION}

\subsection{Deformation}

The procedure for calculating the strains arising in the ironing operation has been described in Section 2.2. The orientation of the maximum principal elongation ratio was calculated for different amounts of reduction. For a reduction of $30 \%$ the average value for $\tan (2 \theta)$ was found to be -0.21 i.e. $\theta=174.07^{\circ}$ rotating anticlockwise or $5.93^{\circ}$ rotating clockwise about the ox axis. Hence the strains can be determined as shown earlier.

\subsection{Texture}

\subsubsection{Cold rolled material}

The complete pole figures for the cold rolled material $(P 2)$ are shown in Figure 6. As expected in an aluminium alloy, the cold rolling texture is similar to the copper

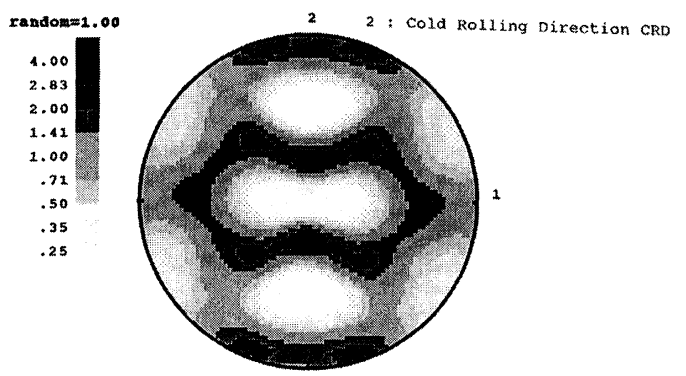

(111)

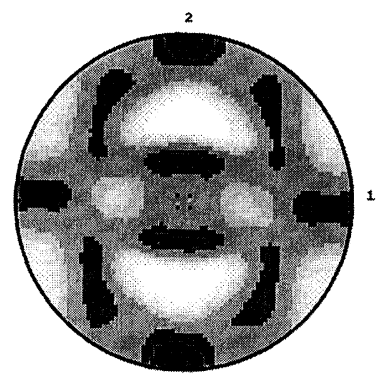

(200)

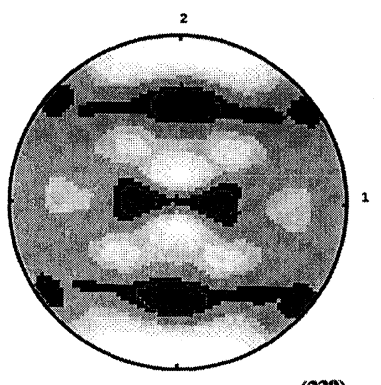

(220)

Figure 6 Pole figures of the as-received material P2. 
rolling texture, which has two strong ideal components i.e. a so called $\mathrm{Cu}$ component - (112)[111], and a S component - (123)[634]. In addition, a remnant of the strong cube texture which had been developed during the hot rolling and annealed process, prior to the cold rolling, can still be detected i.e. Cube component - (001)[100]. The experimental texture after ironing along the transverse direction of the cold rolled material, is shown in Figure 7. The deformed texture is markedly different than the initial texture, in that it is strongly asymmetric. The creation of a stronger Brass component i.e. (110)[112], in the ironed material is also evident.

Figures 8(b) and (c) show the predicted (111) and (200) pole figures obtained from the program LApp, using a full constraints simulation. The ironing process was modelled as plane strain deformation both without and with the inclusion of a shear component. It is evident that by including the shear deformation in the simulaton program gives rise to an asymmetrical texture which agrees very well with that observed experimentally. The experimental pole figures have been shown in Figure 7 but are also given in Figure 8(a) to facilitate the comparison.

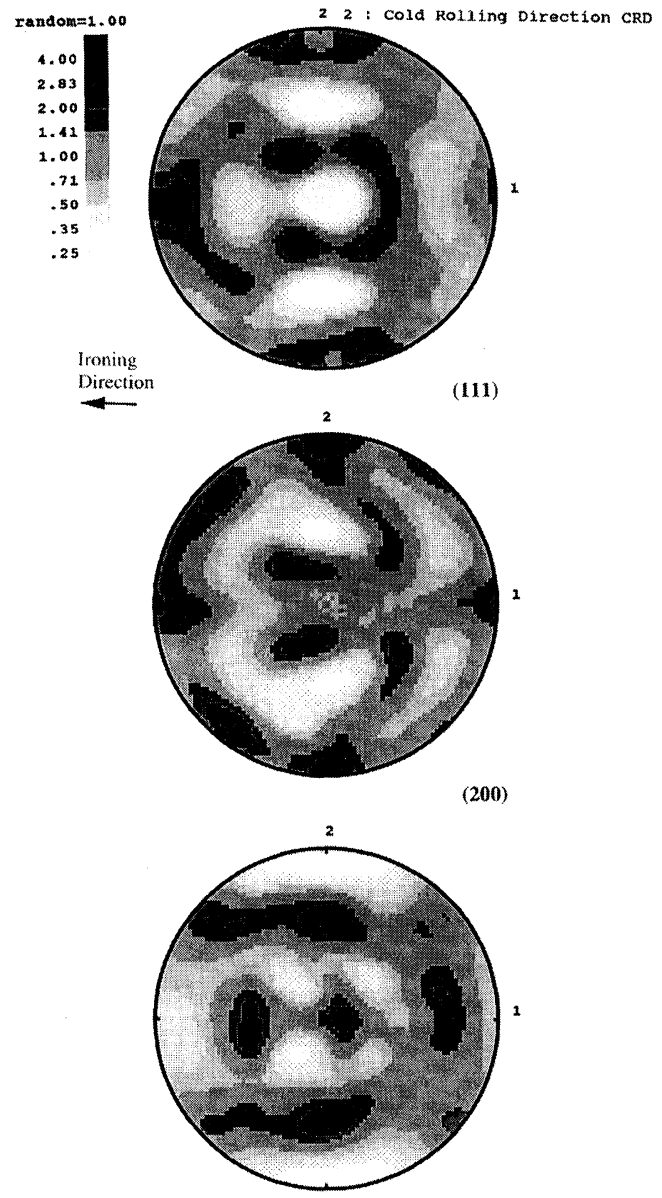

(220)

Figure 7 Pole figures of material P2 after ironing. 


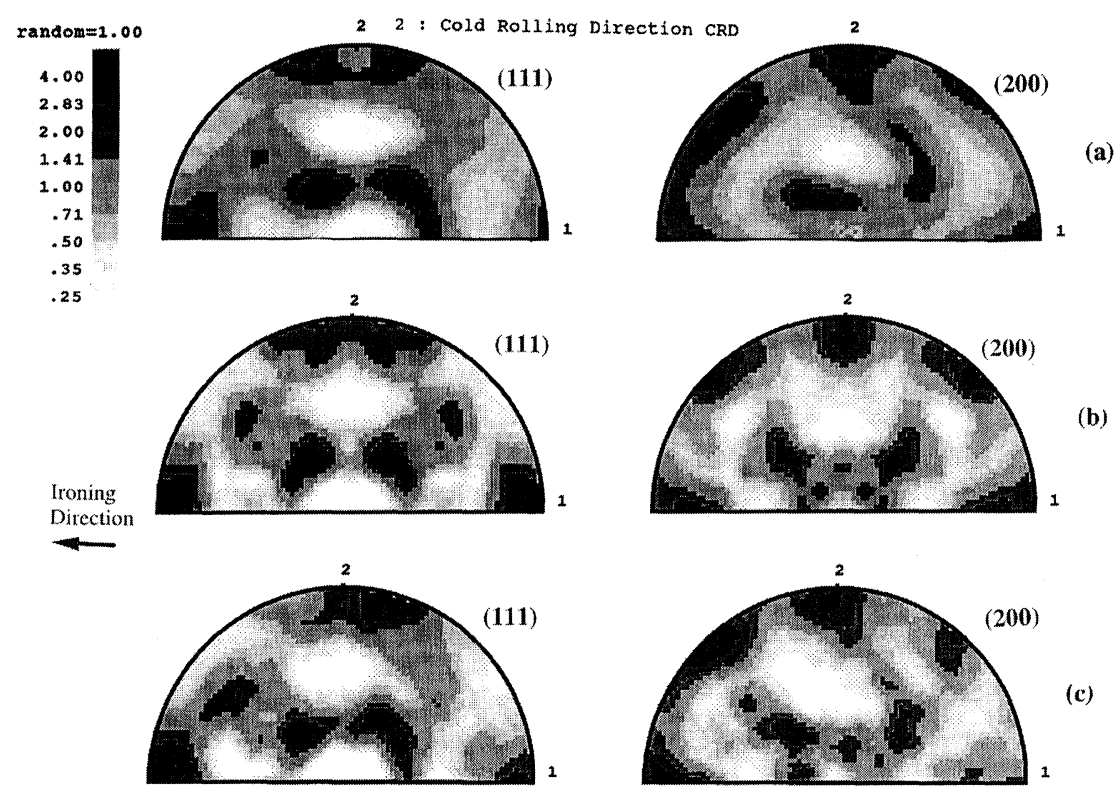

Figure 8 Comparison of the (111) and (200) pole figures for material P2; a) experimental pole figures after ironing. b) predicted pole figures for an axial strain of $32 \%$, but ignoring the shear strain and c) same as (b), but accounting for the shear strain of $\approx 6.9 \%$. The predictions are based on a full constraints simulation with $5^{\circ}$ smoothing.

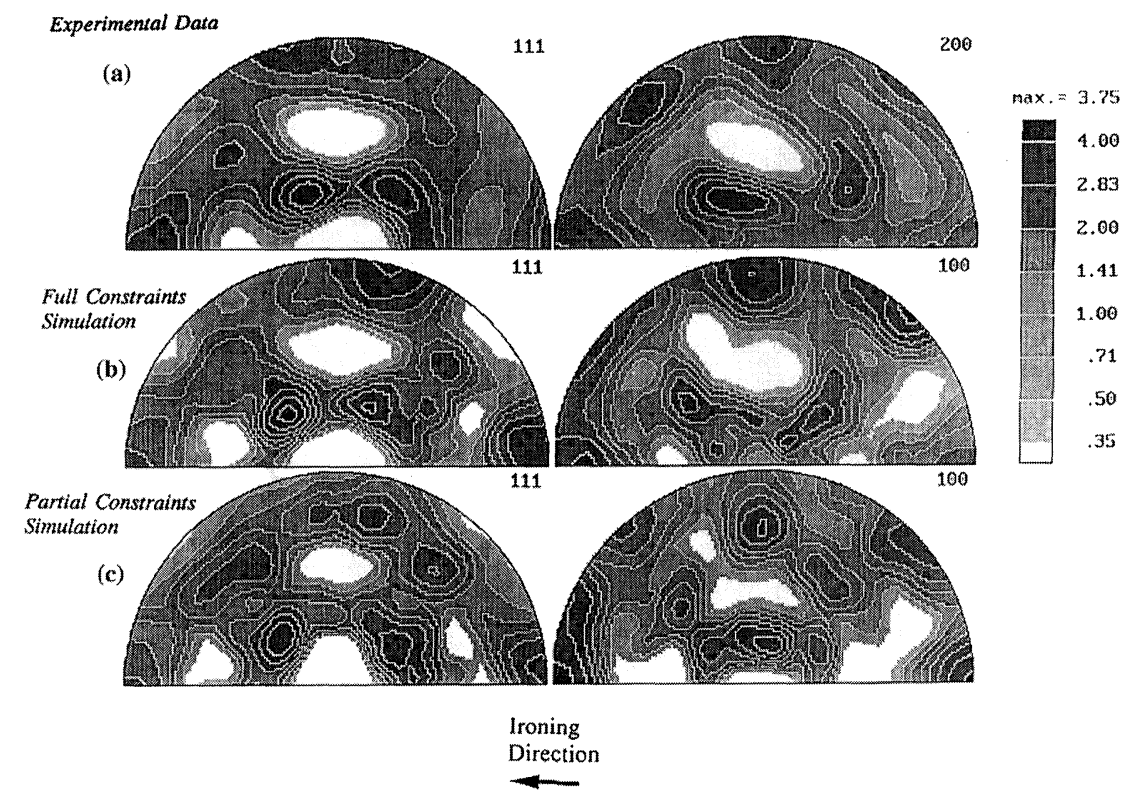

Figure 9 Comparison of the (111) and (200) pole figures for material P2 after ironing. The predictions were performed using a full and partial constraints simulation with $7.5^{\circ}$ smoothing, and allowing for the shear strain; a) experimental pole figures. b) predicted pole figures based on full constraints simulation and c) predicted pole figures based on partial constraints simulation. 
As mentioned at the end of Section 2.3 some additional calculations were performed using a partial constraints simulation (with $7.5^{\circ}$ smoothing), which could also account for the grain aspect ratio, see Table 2. The results of this simulation are shown in Figure 9(c). For comparison Figures $8(\mathrm{a})$ and (c) have been recalculated using $7.5^{\circ}$ smoothing, and are shown in Figures 9(a) and (b) respectively.

\subsubsection{Recrystallized material}

The complete pole figures of the annealed material $(P 2 R)$ are shown in Figure 10. The result is a strong cube texture, associated with a weak remaining cold rolled texture. As pointed out by Mecking (1985) this is the expected texture following the thermalmechanical processing received by the material.

The pole figures following the ironing operation are shown in Figure 11. They are strongly symmetric and similar both in shape and intensity to those shown previously in Figure 7.

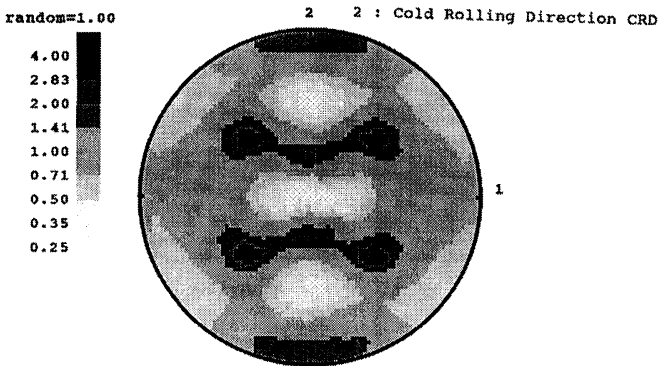

(111)

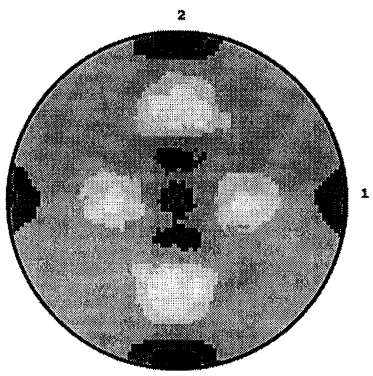

(200)

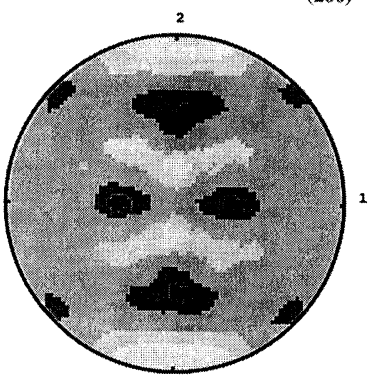

(220)

Figure 10 Pole figures of the recrystallised material $P 2 R$. 


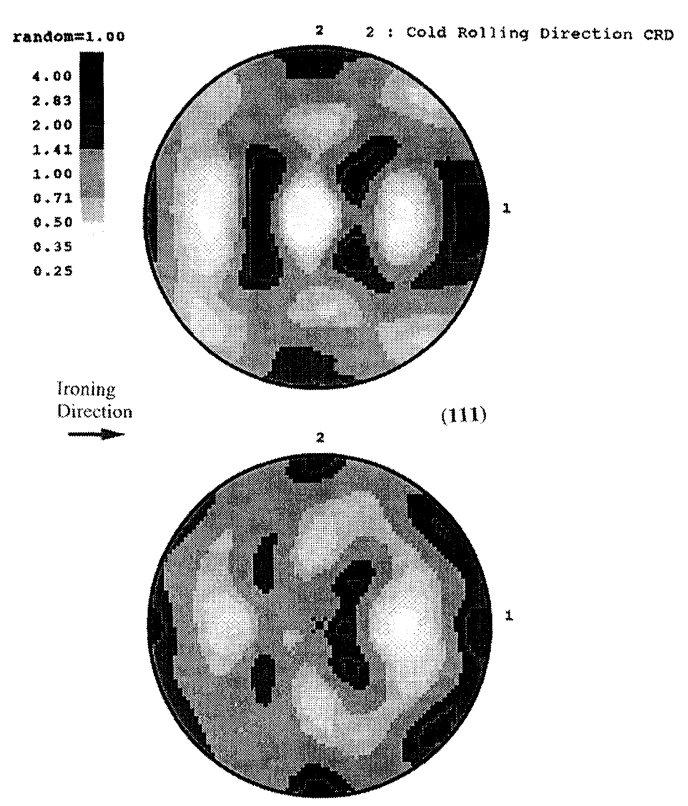

(200)

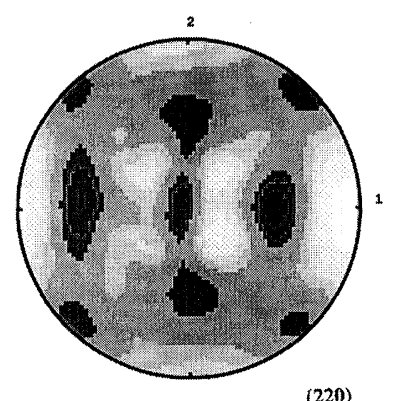

(220)

Figure 11 Pole figures of the material P2R after ironing.

\subsection{Texture Variation through the Thickness}

Texture measurements performed on the materials prior to ironing, did not reveal any difference between the surface and the mid plane, and thus the material was considered homogeneous in the through thickness direction.

The global deformation during ironing which was measured by the use of fiducial marks does not represent the complete deformation path of the metal under the die. The use of a wedged shaped ironing die results in inhomogeneous deformation, with the material being sheared as it enters and leaves the die. In addition there is friction at the die wall and at the surface of the draw bar, and the frictional stresses act in opposite direction. Since the texture evolution is dependant on the total strain path, some difference in texture through the thickness might be expected in the ironed strips. However, no significant change in texture through the thickness was detected in either the cold rolled or the annealed samples after ironing. 


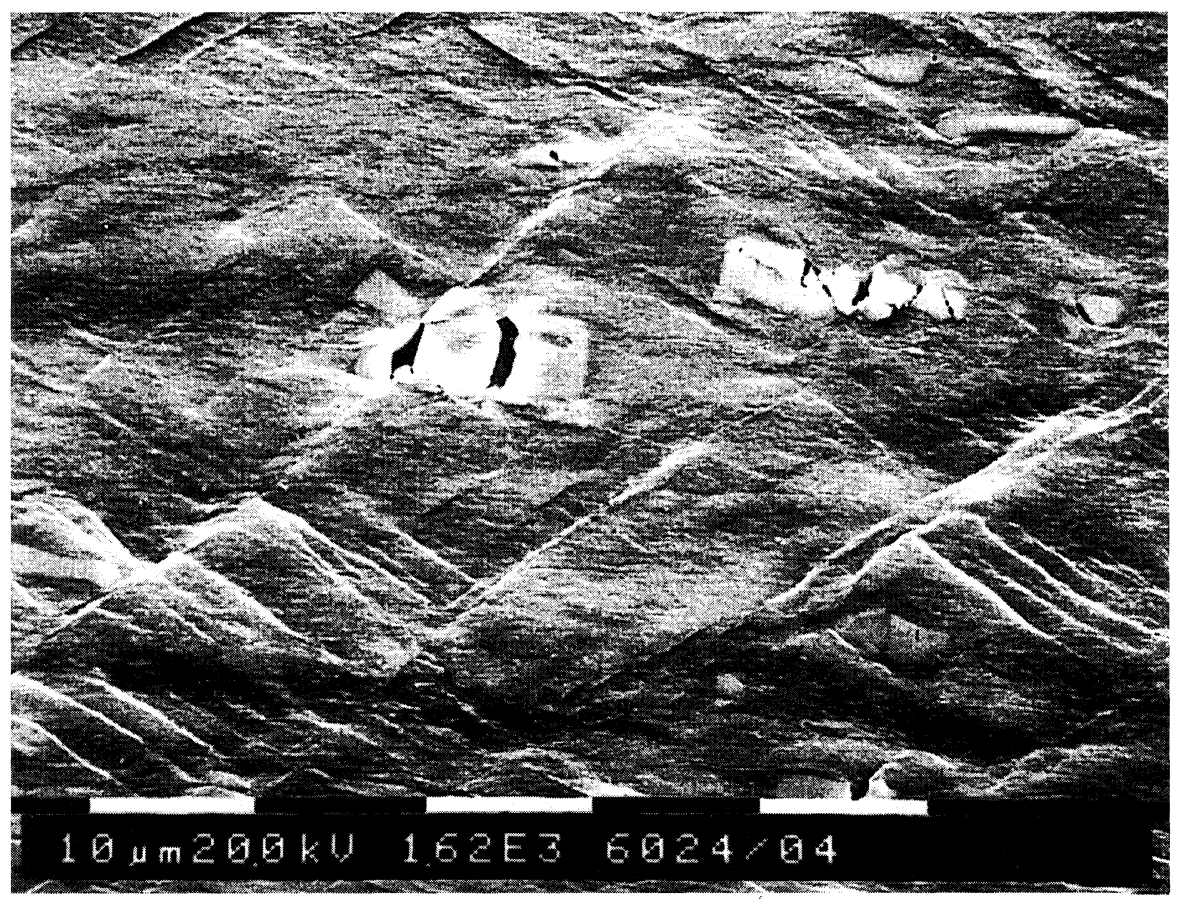

Figure 12 SEM photograph of the through thickness of material P2 after ironing, showing the interaction between the shear bands and the particles.

\subsection{Damage Accumulation}

In addition to the studies of strain path and texture development described above, some observations of damage accumulation were made. The salient features of these observations are that in a number of cases the cracking of $\mathrm{Fe}$-Si inclusions is associated with local shear band development as shown in Figure 12. Also from observations of inclusions in contact with the surface of the ironing die revealed that particle fracture could cause local damage and decohesion which initiate galling as shown in Figure 13.

\section{CONCLUSIONS}

The work reported here shows that an industrial forming process such as ironing can be simulated using a simplified strip drawing operation. Although the simulation does not reproduce a number of important features such as the speed of deformation and the lubrication conditions which exist in the actual forming operation, it does enable the flow process and texture development to be modelled and compared with experimental observations. The texture of the initial sheet material is strongly influenced by the ironing operation, and in both the cold rolled and annealed alloy the measured deformation textures after ironing were similar in appearance and very asymmetric. The texture analysis package, popLA, was quite successful in predicting the experimentally 


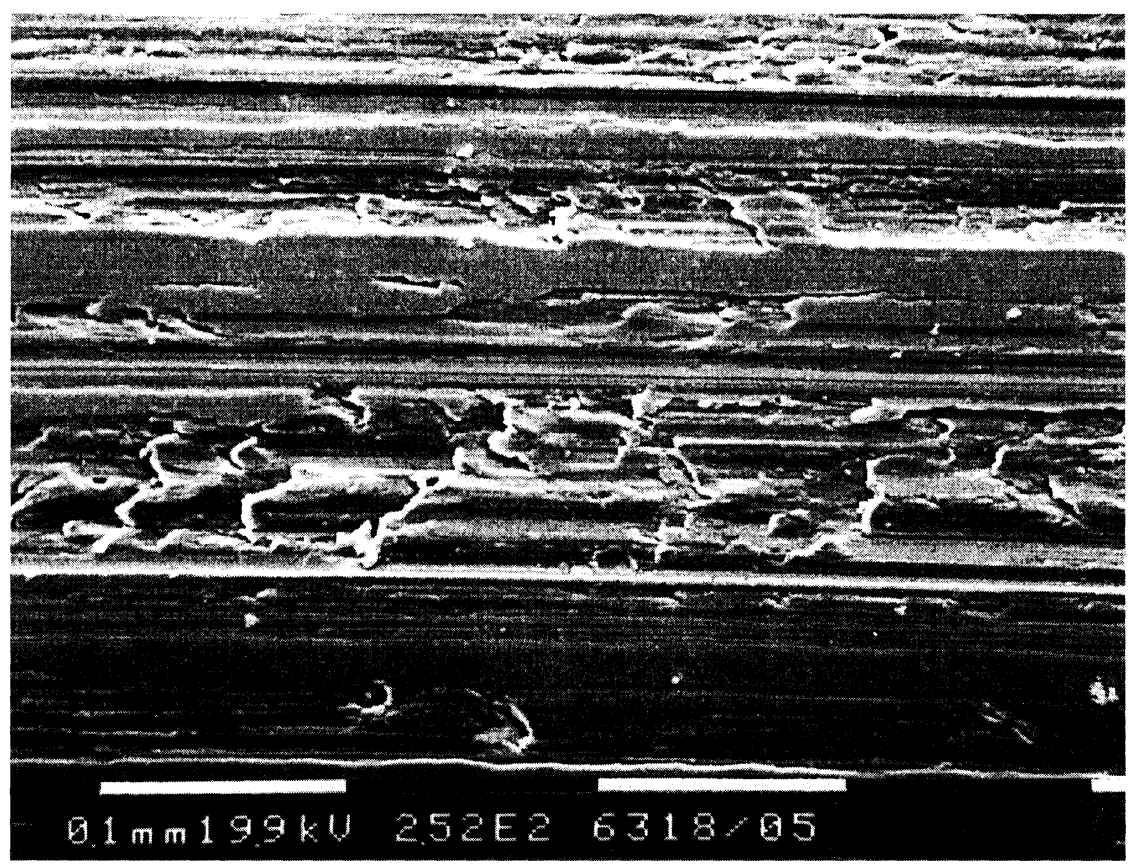

Figure 13 SEM photograph of the surface of material P2 showing galling after ironing.

observed textures. Of particular interest was the prediction of the texture evolution for the cold rolled material using both a full and partial constraints simulation. The latter method could account for the fact that the grains were not equiaxed.

The metallographic studies performed as part of this work revealed information about grain shape, local deformation modes and damage events, which are of value in understanding the structural changes which arise in the ironing process.

\section{Acknowledgements}

The authors would like to thank the Natural Sciences and Engineering and Research Council of Canada, the Manufacturing Research Corporation of Ontario and the Ontario Centre for Materials Research for financial support, and the technical input from some of the research staff at the Aluminium Company of Canada (H. Sang and G. B. Burger), Pechiney CRV (J. Courbon) and the Los Alamos National Laboratories. The work at Los Alamos was sponsored by the US Department of Energy. Thanks also to Mr. J. Nougier (Attache Scientifique, Ambassade de France au Canada) for arranging for one of us (FH) to study in Canada under the French VSNA programme.

\section{References}

Heymès, F., (1992), M. Eng. Thesis, Department of Mechanical Engineering, McMaster University, Hamilton, Ontario, Canada.

Hone, A. and Pearson, E. C., (1950), "A New Anodic-Film Method for Studying Orientation in Aluminium”, Metal Progress, Vol. 50, pp. 713-715. 
Kallend, J. S., Kocks, U. F., Rollett, A. D. and Wenk, H. R., (1991), “Operational Texture Analysis”, Materials Science and Engineering Analysis, Vol. A132, pp. 1-22.

Kocks, U. F., Canova, G. R., Tomè,, C. N. and Rollett, A. D., (1988), "Los Alamos Polycrystal Plasticity Code", Los Alamos Software Package LA-CC-88-6.

Mecking, H., (1985), "Texture in Metals" in Preferred Orientation in Deformed Metals and Rocks: an Introduction to Modern Texture Analysis, (Editor, Hans-Rudolf Wenk), Academic Press.

Sowerby, R., Duncan, J. L. and Chu, E., (1986), "The Modelling of Sheet Metal Stampings", Int. J. Mech. Sciences, Vol. 28, pp. 415-430.

Sowerby, R. and Chakravarti, P. C., (1983), "The Determination of Equivalent Strain in Finite Homogeneous Deformation Processes”, J. Strain Analysis, Vol. 18, pp. 119-123. 